\title{
Screening and treatment of tuberculosis among pregnant women in Stockholm, Sweden, 2016-2017
}

\author{
Gabrielle Fröberg $\mathbb{1}^{1,2}$, Lena Jansson ${ }^{1}$, Katherine Nyberg ${ }^{1}$, Birgitta Obasi ${ }^{3}$, \\ Katarina Westling ${ }^{1,4}$, Ingela Berggren ${ }^{5}$ and Judith Bruchfeld ${ }^{1,2}$
}

Affiliations: 'Dept of Infectious Diseases, Karolinska University Hospital, Stockholm, Sweden. ${ }^{2}$ Division of Infectious Diseases, Dept of Medicine Solna, Karolinska Institutet, Stockholm, Sweden. ${ }^{3}$ Unit of Maternal Health Care, Dept of Women's Health, Södersjukhuset, Stockholm, Sweden. 'Division of Infectious Diseases and Dermatology, Dept of Medicine Huddinge, Karolinska Institutet, Stockholm, Sweden. ${ }^{5}$ Dept of Communicable Diseases Control and Prevention, Stockholm County Council, Stockholm, Sweden.

Correspondence: Gabrielle Fröberg, B3:00, Dept of Infectious Diseases, Karolinska University Hospital, 17176 Stockholm, Sweden. E-mail: gabrielle.frobergdill.se

@ERSpublications

Systematic TB screening of pregnant women in Stockholm was feasible with high yield of unknown latent TB and mostly asymptomatic active TB. Optimised routines improved referrals to specialist care. Adherence to treatment of latent TB was very high. http://bit.ly/2NrhEwk

Cite this article as: Fröberg G, Jansson L, Nyberg K, et al. Screening and treatment of tuberculosis among pregnant women in Stockholm, Sweden, 2016-2017. Eur Respir J 2020; 55: 1900851 [https://doi.org/ 10.1183/13993003.00851-2019].

ABSTRACT Swedish National tuberculosis (TB) guidelines recommend screening of active and latent TB (LTBI) among pregnant women (PW) from high-endemic countries or with previous exposure to possibly improve early detection and treatment.

We evaluated cascade of care of a newly introduced TB screening programme of pregnant women in Stockholm county in 2016-2017. The algorithm included clinical data and Quantiferon (QFT) at the Maternal Health Care clinics and referral for specialist care upon positive test or TB symptoms.

About $29000 \mathrm{HIV}$-negative pregnant women were registered yearly, of whom $11 \%$ originated from high-endemic countries. In 2016, 72\% of these were screened with QFT, of which $22 \%$ were QFT positive and $85 \%$ were referred for specialist care. In 2017, corresponding figures were $64 \%, 19 \%$ and $96 \%$, respectively. The LTBI treatment rate among all QFT-positive pregnant women increased from $24 \%$ to $37 \%$ over time. Treatment completion with mainly rifampicin post-partum was $94 \%$. Of the 69 registered HIV-positive pregnant women, 78\% originated from high-endemic countries. Of these, $72 \%$ where screened with QFT and 15\% were positive, but none was treated for LTBI. 9 HIV-negative active pulmonary TB cases were detected (incidence: 215/100000). None had been screened for TB prior to pregnancy and only one had sought care due to symptoms.

Systematic TB screening of pregnant women in Stockholm was feasible with a high yield of unknown LTBI and mostly asymptomatic active TB. Optimised routines improved referrals to specialist care. Treatment completion of LTBI was very high. Our findings justify TB screening of this risk group for early detection and treatment. 


\section{Introduction}

Pregnancy is a state of relative immunosuppression characterised by anti-inflammatory cellular responses that promote tolerance to fetal antigens. Pregnancy induces a down regulation of the T-helper type 1 (Th1) immunity leading to impaired cell-mediated immunity with decreased levels of interferon (IFN)- $\gamma$ and tumour necrosis factor (TNF)- $\alpha$ and therefore a theoretically increased susceptibility to activation and/or de novo infections of certain intracellular microbes such as Mycobacterium tuberculosis [1, 2]. Quiescent or subclinical tuberculosis (TB) with few and non-specific symptoms is more common during pregnancy with an abrupt onset of a pro-inflammatory response after delivery which may lead to overt clinical manifestations, i.e. immune reconstitution syndrome (IRS) [3, 4]. Pregnancy and, in particular, the post-partum period have been debated as linked to a higher risk of active TB and contributors to both maternal, fetal and newborn morbidity and mortality, especially among HIV-positive women [5-11].

As part of the global End TB strategy [12], screening and treatment of latent TB infection (LTBI) is now recommended in low-endemic middle- and high-income countries for certain risk groups, such as immunosuppressed individuals from high-endemic countries $[13,14]$. In line with this strategy, the Public Health Agency of Sweden recommends screening for LTBI among pregnant women from high-TB endemic countries (incidence $\geqslant 100 / 100000$ ) or exposed to known/suspected contagious TB [15]. In Sweden, asylum seekers are offered a health examination upon arrival, including TB screening for high-risk groups. However, coverage is only about 50\% [16] and other migrants, such as reunification family members, are only entitled to a health examination upon their own request, while other immigrants, e.g. labour immigrants and European Union (EU) migrants are not entitled to examination. Therefore, many pregnant women from high-endemic countries have not been screened for TB after arrival in Sweden and routine check-ups at the Maternal Health Care (MHC) clinics therefore offer a good opportunity to screen for unknown TB in this group.

In January 2016, Stockholm county introduced systematic screening of both active TB and LTBI among all pregnant women from high-endemic countries (figure 1) in collaboration between MHCs, the Department of Communicable Diseases Control and Prevention at Stockholm County Council and the TB centre and HIV centre at Department of Infectious Diseases, Karolinska University Hospital (Karolinska, Sweden). QFT was preferred before the standard tuberculin test (TST) because of the advantage of only one visit needed for testing, as well as better performance to detect assumed LTBI during pregnancy [17-21]. Treatment of LTBI was initiated after delivery (if not recently exposed to contagious TB) [15] to minimise the risk of adverse events during pregnancy. Shorter LTBI treatments such as rifampicin (RIF) once daily (OD) for 4 months (4R), isoniazide (INH) plus RIF OD for 3 months (3HR) and high-dose INH and rifapentine (RPT) once weekly for 12 weeks (3HP) have been shown to be as efficacious and safe as INH with pyridoxine substitution OD for 9 months $(9 \mathrm{H})$ and significantly associated with increased completion rates [22, 23]. 3HP is at present not a validated option in pregnancy or breast feeding; however, trials are on-going (www.clingovtrials.com). TB care and treatment is free of charge according to the Swedish Communicable Disease Act.

Our objective was to evaluate cascade of care of the newly introduced TB screening programme among pregnant women in Stockholm in 2016-2017.

\section{Material and methods}

This was a retrospective, observational study of pregnant women screened for active TB and LTBI in Stockholm during 2016 and 2017. HIV-negative pregnant women were screened at the regular MHC clinics in Stockholm and referred to the centralised TB centre at Karolinska upon a positive screening result (figure 1). HIV-positive pregnant women were screened and followed up at the specialist MHC clinic and the HIV centre at Karolinska.

At MHC, mainly during the first trimester, information was registered on country of birth, year of arrival to Sweden, previous history and/or contact with active TB, previous testing and/or treatment for LTBI and symptoms of active TB, i.e. fever, night sweats, weight loss and/or cough. If symptoms of active TB were detected, the subject was immediately referred to the TB centre at Karolinska for diagnostic procedures according to routine clinical practice. Asymptomatic pregnant women from high-endemic countries or with known TB exposure were tested with QFT and analysis was performed according to the manufacturer's instructions at the Karolinska TB laboratory. If QFT was negative $\left(<0.35 \mathrm{IU} \cdot \mathrm{mL}^{-1}\right)$, information was given to the subject by the midwife. If the QFT was positive $\left(\geqslant 0.35 \mathrm{IU} \cdot \mathrm{mL}^{-1}\right)$, information was given to the subject at an appointment with an MHC physician and thereafter referred for chest radiograph (CXR) and then specialist care.

In June 2016, some adjustments were introduced to the screening criteria as a consequence of QFT-positive pregnant women referred for specialist care, but who, after assessment, were not recommended treatment. Adjustments were made regarding QFT which was not recommended if the subject: a) had been previously 


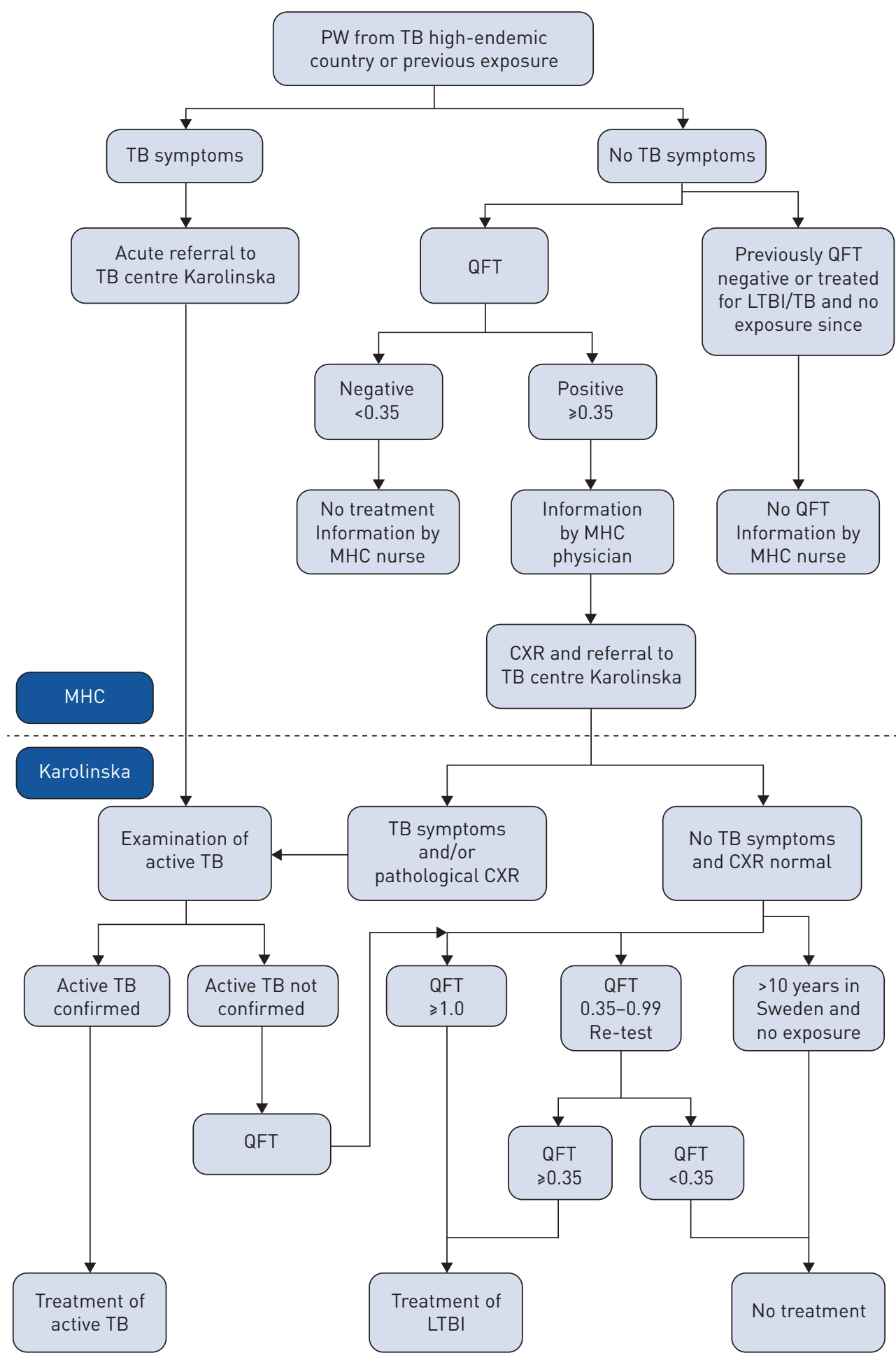

FIGURE 1 Flow chart of tuberculosis (TB) screening algorithm for pregnant women (PW) from high-endemic countries or previous exposure. QFT: Quantiferon test; MHC: Maternal Health Care.

tested TST-/QFT-negative and not been exposed to TB thereafter; or b) had been previously treated for LTBI or active TB (but still referred if exposed to contagious TB thereafter) [24]. Further, referrals to CXR and specialist care were recommended in parallel, as some pregnant women had been unwilling to perform CXR with delayed/absent referral to specialist care as a consequence.

Referred pregnant women with symptoms and/or abnormal CXR were seen by a specialist/resident physician in infectious diseases within 1 week for further diagnostic work-up. Asymptomatic pregnant 
women with normal CXR were seen within 4 weeks, mainly during the second trimester. Pregnant women with a QFT borderline-positive result $\left(0.35-0.99 \mathrm{IU} \cdot \mathrm{mL}^{-1}\right)$ or indeterminate result were re-tested [25]. Pregnant women with no CXR performed were thoroughly informed by the physician about the harmlessness and importance of the examination.

Treatment of LTBI was initially recommended if the subject was $<30$ years old and had migrated to Sweden within 2 years; or visited her home country or another high-endemic country for $>3$ months in the previous 2 years; or was previously exposed to contagious TB within 2 years; or had co-morbidity with a higher risk of TB activation, such as severe diabetes mellitus, kidney failure, underweight or immunosuppressive treatment; or pathological CXR indicating previous TB (and where active TB was excluded). In June 2016, the age-limit was removed as criteria and the limit of $>2$ years in Sweden or since exposure was adjusted to $>10$ years, due to several cases of active TB among pregnant and post-partum women aged $>30$ years with arrival to Sweden $>2$ years ago.

All pregnant women were planned to initiate LTBI treatment within 1 month after delivery. To minimise loss to follow-up, pregnant women recommended for treatment were scheduled for an appointment with a physician 1-2 weeks after the estimated time of delivery and a letter to the midwife with LTBI information and instructions to contact the TB centre after delivery. Pregnant women exposed to contagious TB recently (<2 years) started LTBI treatment already during pregnancy. During treatment, the pregnant women had check-up appointments with a TB nurse at 2 weeks after initiation and then usually every month. Adherence was assumed if self-reported and if the woman complied with the monitoring including blood tests for liver enzymes. Severe adverse events or other concerns were reported to the physician for assessment. First-line treatment of LTBI for pregnant and post-partum women during the study period was $4 \mathrm{R}$ with $3 \mathrm{HP}$ as an alternative for post-partum women who did not breast feed. Pregnant women with CXR infiltrates without activity were recommended $9 \mathrm{H}$.

The study included only ordinary management procedures covered by the regular patient insurance. All compiled data were anonymised to obtain minimal integrity intrusion for the patient. MHC data was collected and compiled by the Department of Communicable Diseases Control and Prevention, Stockholm County Council, and then stored together with data from the specialised care in a safe database at Karolinska for centralised analyses (table 1). Ethical permission was granted from Stockholm Regional Ethics committee (Dnr 2018/555-31) who waivered the necessity of informed consent.

\section{Results}

Screening and treatment of LTBI among HIV-negative pregnant women

In 2016, a total of 29459 pregnant women were registered in Stockholm (table 1); 3054 (10\%) originated from high-TB endemic countries and, of these, 2184 (72\%) were screened with QFT at the regular MHC clinics. Of the screened pregnant women, 479 (22\%) were QFT positive, which is in line with estimated LTBI prevalence in high-endemic countries [14]. Of these, 407 (85\%) were referred to the TB centre at Karolinska.

In 2017, the corresponding figures were 28805 pregnant women, 3140 (11\%) originated from high-endemic countries, 1994 (64\%) were screened with QFT, 383 (19\%) were QFT positive and 367 (96\%) were referred.

TABLE 1 Yield of screening and treatment of latent tuberculosis (LTBI) among HIV-negative and HIV-positive pregnant women in Stockholm, 2016-2017

\begin{tabular}{lccc} 
& $\mathbf{2 0 1 6}$ & $\mathbf{2 0 1 7}$ & HIV 2016-2017 \\
\hline Pregnant women total $^{\#}$ & 29459 & 28805 & 69 \\
High endemic origin $^{\#}$ & $3054(10)$ & $3140(11)$ & $54(78)$ \\
Screened with QFT $^{\pi}$ & $2184(72)$ & $1994(64)$ & $39(72)$ \\
QFT positive $^{\pi}$ & $479(22)$ & $383(19)$ & $6(15)$ \\
Referred $^{+}$ & $407(85)$ & $367(96)$ & na \\
Initiated LTBI treatment $^{+}$ & $113(28) ;(24 \%$ of & $142(39) ;(37 \%$ of & 0 \\
Completed LTBI $_{\quad}$ & QFT-positive) & QFT-positive) & \\
treatment $^{+}$ & $106(94) ;(22 \%$ of & $128(90) ;(33 \%$ of & na \\
& QFT-positive) & QFT-positive) &
\end{tabular}

Data are presented as n or $\mathrm{n}(\%)$, unless otherwise stated. QFT: Quantiferon test; na: not applicable. Data collected by "Department of Growth and Regional Planning Stockholm County Council, "Karolinska University Laboratory and ${ }^{+}$TB centre, Department of Infectious Diseases, Karolinska University Hospital. 
Referred pregnant women who initiated treatment increased from 113 (28\%) out of 407 in 2016 to 142 (39\%) out of 367 in 2017 (table 1). Upon the physicians' decision, 109 (21\%) of all women were not recommended treatment due to previous treatment of TB/LTBI, 93 (18\%) due to migration to Sweden $>10$ years ago, 45 (9\%) due to negative QFT result upon retesting and 29 (6\%) due to other causes, e.g. miscarriage/abortion, frequent travels to high-risk countries, MDR exposure or elevated liver enzymes. In addition, in June 2016, the inclusion criteria for treatment was adjusted as described previously and, therefore, $83(16 \%)$ women were not recommended treatment due to age $>30$ years and/or arrival to Sweden $>2$ years ago. Furthermore, 22 (4\%) women emigrated/moved, 38 (7\%) were unwilling to undergo treatment and/or were lost to follow-up. In total, the proportion of all QFT-positive pregnant women that initiated LTBI treatment increased from 113 (24\%) out of 479 in 2016 to 142 (37\%) out of 383 in 2017. In total, six pregnant women started treatment during pregnancy, while the remaining started after delivery.

A total of 255 women initiated LTBI treatment in 2016-2017 (table 2). Five women still on treatment (3 RIF and 2 INH) were excluded from further analyses. The mean (range) age was 30.1 (20-41) years and time in Sweden was $4.9(0-31)$ years. In summary, 156 (62\%) pregnant women originated from Africa, 86 (34\%) from Asia, seven (3\%) from Europe and $<1 \%$ from America. The majority, 224 (90\%), was treated with $4 \mathrm{R}$ and remaining with $9 \mathrm{H}, 3 \mathrm{HR}$ or $3 \mathrm{HP}$.

A total of 234 (94\%) women completed treatment, while remaining discontinued due to adverse events (6 out of 16), re-location (3 out of 16) or loss to follow-up (7 out of 16).

Cascade of care for all HIV-negative pregnant women intended for screening is presented in figure 2.

\section{Screening and treatment of LTBI among HIV-positive pregnant women}

A total of 69 HIV-positive pregnant women were registered in Stockholm in 2016-2017 (table 1), 54 (78\%) originated from TB high-endemic countries, of those 59 (72\%) were screened with QFT and of these six (15\%) were QFT positive, but with no clinical or radiological signs of TB. None was treated for LTBI. One subject started treatment for suspected lymph node TB but was instead diagnosed with lymphoma, two had elevated liver enzymes due to chronic hepatitis, one had a legal abortion and two did not start due to prolonged travels abroad.

\section{Detection and treatment of active TB}

A total of nine microbiologically confirmed active pulmonary TB cases were detected during pregnancy or post-partum (table 3). All were HIV negative. The mean (range) age was 29.2 (23-33) years. Five had their first child. Seven originated from high-endemic countries and time in Sweden was 4.2 (1-12) years. None had been screened for TB after arrival to Sweden. Five had previously been exposed to TB and two had been treated for active TB previously, but one of them had discontinued treatment after 4 months.

Six of the active TB cases were referred to Karolinska from MHC via the screening programme and were diagnosed in the second trimester. Of the remaining three cases, one case had not been included in the MHC screening due to uncertain migration status and was detected post-partum in asylum screening. One case had been abroad during pregnancy and was detected post-partum when seeking primary health care

TABLE 2 Outcome of treatment of latent tuberculosis infection (LTBI) among HIV-negative women in 2016 and 2017

\begin{tabular}{lccc} 
LTBI treatment & $\mathbf{2 0 1 6}$ & $\mathbf{2 0 1 7}$ & Total \\
\hline Completed & $106(94)$ & $128(93)$ & $234(94)$ \\
RIF & $86(95)$ & $124(93)$ & $210(94)$ \\
INH & $7(78)$ & $3(100)$ & $10(83)$ \\
INH+RIF & $12(100)$ & $1(100)$ & $13(100)$ \\
INH+RPT & $1(100)$ & 0 & $1(100)$ \\
Discontinued & $7(6)$ & $9(7)$ & $16(6)$ \\
RIF & $5(5)$ & $9(7)$ & $14(6)$ \\
INH & $2(22)$ & $0(0)$ & $2(17)$ \\
INH+RIF & 0 & 0 & 0 \\
INH+RPT & 0 & 0 & 0 \\
Total & 113 & 137 & 250
\end{tabular}

Data are presented as $\mathrm{n}(\%)$ or $\mathrm{n}$. Proportion (\%) that completed or discontinued of all treated as well as for each respective drug. RIF: rifampicin; INH: isoniazid; RPT: rifapentine. 


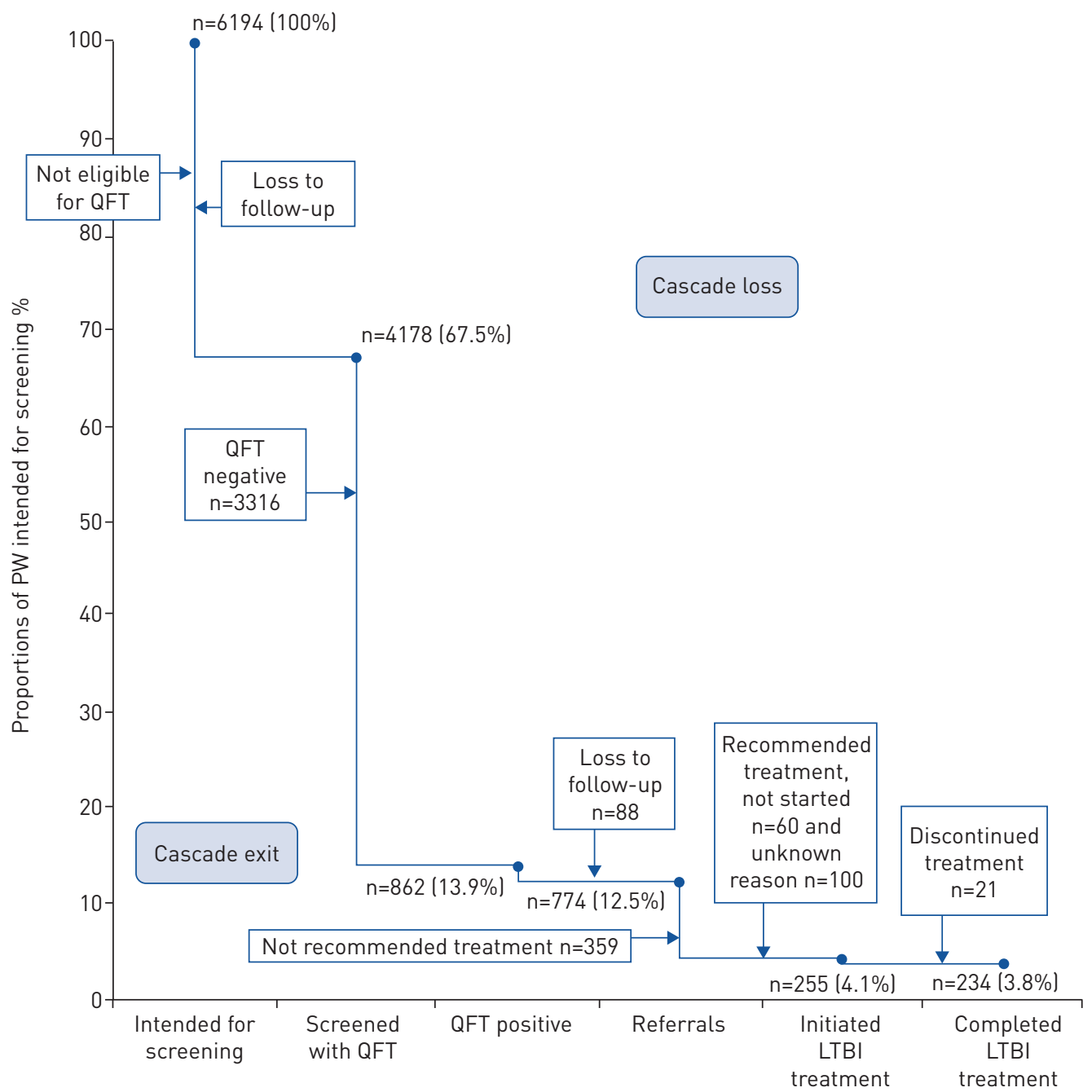

FIGURE 2 Proportions (\%) of all HIV-negative pregnant women (PW) from high-endemic countries (intended for screeningl through the cascade of care in Stockholm county, 2016-2017.

TABLE 3 Details of active tuberculosis (TB) cases detected during pregnancy or post-partum in Stockholm 2016-2017

\begin{tabular}{|c|c|c|c|c|c|c|c|c|c|c|}
\hline Age years & Parity n & Origin/TB incidence & $\begin{array}{l}\text { Years in } \\
\text { Sweden }\end{array}$ & $\begin{array}{c}\text { Previous } \\
\text { TB expo/tx }\end{array}$ & Symptoms & QFT IU.mL-1 & CXR & $\begin{array}{c}\text { Sputum } \\
\text { [SM/PCR/culture] }\end{array}$ & GL/BAL & Diagnosis \\
\hline 29 & 2 & Africa/high & 2 & $-1-$ & No & 3.48 & PE & $-/-1+$ & & PTB (S) \\
\hline 33 & 1 & Asia/high & 6 & $+/-$ & No & 2.13 & Normal & $-/-1+$ & & PTB (PZA-R) \\
\hline 23 & 1 & Europe/middle & 1 & $+1-$ & No & $0.56 / 2.69$ & $\mathrm{PE}+\mathrm{Al}$ & $-1-1-$ & $+/+$ & PTB (S) \\
\hline 33 & 3 & Africa/very high & 3 & $+/-$ & No & 5.53 & $\mathrm{Al}$ & $-/-1+$ & +1 & PTB (S) \\
\hline 29 & 1 & Africa/high & 1 & $-1-$ & Slight cough & 3.50 & Normal & $-1-1+$ & & PTB (S) \\
\hline 27 & 1 & Asia/high & 1 & $-/+$ & No & 9.83 & $\mathrm{PE}+\mathrm{Al}$ & $-/+/+$ & +1 & PTB (RIF-R) \\
\hline 33 & 3 & Europe/low & 7 & $-1-$ & Cough, weight loss & na & Al+cavity & $+/+/+$ & & PTB (S) \\
\hline 33 & 3 & Asia/high & 5 & $+1-$ & No & 2.04 & $\mathrm{Al}$ & $-/-1+$ & +1 & PTB (MDR) \\
\hline 23 & 1 & Asia/high & 12 & $+/+$ & Slight cough & 2.90 & $\mathrm{Al}$ & $-/+/+$ & -1 & $\mathrm{PTB}+\mathrm{LNTB}(\mathrm{S})$ \\
\hline
\end{tabular}

Expo: exposure; tx: treatment; QFT: Quantiferon test; CXR: chest radiograph; SM: smear microscopy; GL: gastric lavage; BAL: broncho-alveolar lavage; PE: pleural effusion; Al: apical infiltrate; PTB: pulmonary TB; LNTB: lymph node TB; S: sensitive TB; PZA-R: Pyrazinamide resistant TB; RIF-R: Rifampicin resistant TB; MDR: multi-drug resistant TB; na: not applicable. TB incidence: high >100/100000, middle 25-100/100000, low <25/100000; Generally, standard treatment was given for 6 months or prolonged to 9-12 months for mono-resistant cases. The MDR-TB case has been on adjusted regiments due to different adverse events. 
for prolonged cough and weight loss. One case was detected in contact tracing at Karolinska and at the same time diagnosed as pregnant in the first trimester.

Only one of the pregnant women with active disease had experienced symptoms associated with TB and two reported slight cough when questioned for symptoms, while the remaining women were asymptomatic. Eight were tested with QFT and all were positive (range 2.13-9.83 IU $\cdot \mathrm{mL}^{-1}$ ). Seven had pathological CXRs with infiltrates and/or pleural effusions. The symptomatic case had cavitary upper lobe infiltration and was smear microscopy positive in sputum, while the remaining were smear microscopy negative. Three were PCR positive and all were M. tuberculosis culture verified in sputum and/or gastric/ bronchoalveolar lavage.

Six patients had drug-sensitive TB. Two patients had mono-resistant TB i.e. RIF and Pyrazinamide (PZA), respectively. One patient had multi-drug resistant (MDR) TB.

\section{Discussion}

We evaluated cascade of care of the recently introduced TB screening programme among pregnant women in Stockholm in 2016 and 2017 (figure 2). The choice of TB screening among pregnant women originating from countries with an incidence of $>100 / 100000$ is in line with recent reviews by PAREEK et al. [26] and GreEnaway et al. [27] in which screening of LTBI in migrants 16-35 years old and originating from countries with a TB incidence of $>150 / 100000$ was the most cost-effective strategy to prevent one active TB case.

In June 2016, several adjustments in the recommendations for screening, referral and LTBI treatment were introduced as described previously. This adjustment resulted in fewer pregnant women eligible for QFT and thus probably a "false" higher drop-off in the cascade of care from "intended for screening" to "QFT screened". However, we do not have information on these figures but according to the MHC nurses, unwillingness by pregnant women to undergo QFT and/or loss to follow-up was uncommon. Furthermore, parallel referrals to CXR and to specialist care markedly decreased drop-off from "QFT positives" to "referred". The wider recommendations for screening as well as the more narrow inclusion criteria for LTBI treatment before June 2016 resulted in many pregnant women not recommended for treatment upon specialist decision and a rather large drop-off from "referrals" to "initiated treatment".

However, these drop-offs were smaller than in a recent meta-analysis by ALsDuRf et al. [28], in which the proportion of eligible individuals that completed the different steps in the cascade of care was highly heterogeneous between identified risk groups. For migrants, only $43 \%$ completed testing and $14 \%$ of eligible individuals completed treatment. Corresponding figures among HIV-negative pregnant women in our study were $68 \%$ and $27 \%$, respectively.

Earlier identified difficulties in communicating a positive test result for LTBI, not being the same as active TB [29], was confirmed resulting in unnecessary anxiety among pregnant women. Knowledge and perceptions improved with continued information and feed-back to the screening units [30].

Treatment for LTBI was generally well tolerated with a high completion rate of $94 \%$. Adherence was, however, not directly observed, but assumed if self-reported and fulfilled follow-up, which might be a limitation. Only $6 \%$ of patients treated with $4 \mathrm{R}$ discontinued and none discontinued with $3 \mathrm{HR}$, while $17 \%$ of patients treated with $9 \mathrm{H}$ discontinued, which is in line with previous studies reporting higher completion rates with shorter LTBI treatments $[22,23]$. All but six pregnant women initiated treatment after delivery. However, relative immune impairment and a possible quiescent TB activation during pregnancy may suggest that treatment should be initiated before delivery.

Among the HIV-positive pregnant women, a higher proportion originated from high-endemic countries compared with HIV-negative pregnant women (78 versus 11\%). The proportion of QFT-positive pregnant women was $15 \%$ and not significantly different from HIV-negative pregnant women. However, KoNIG WALLES et al. [18] observed lower levels of IFN- $\gamma$ in HIV-positive pregnant women compared with HIV-negative pregnant women. This may indicate a higher risk of false negative QFT results among HIV-positive pregnant women with LTBI. In a recent report by NorRBY et al. [31], the incidence of active TB among HIV-positive subjects was 80 -times higher than the general population in Stockholm, which emphasises the importance of TB screening and treatment of pregnant women in this group.

In this study, MHC-based screening as a complement to the regular asylum screening, found a high yield of active TB. This is supported by KUNST et al. [32], in which median yield was 431/100000 among 21 studies reporting on post-arrival community-based screening of migrants. This can be compared with 93/100000 for pre-entry screening, 29/100000 for screening at port of arrival and 119/100000 for screening at reception centres. Furthermore, active TB among pregnant women is a high priority as it also include a vulnerable fetus/neonate, which is not the case for migrants in general. However, coverage was 
lower for community-based screening, compared with pre- or peri-migration screening (64\% versus $93 \%$ ), which is in line with the regular asylum screening in Sweden [16] and is also reflected in the fact that none of the active TB cases in this study had been screened for TB after arrival to Sweden.

GrEenAWAY et al. [27], showed that screening with CXR for active TB among migrants was highly sensitive (98\%) but only moderately specific (75\%). Yield results varied widely depending on country of origin, migrant type and screening setting. The highest yield of 336/100000 screened was seen among migrants originating from very high TB incidence countries $(>350 / 100000)$, while for high-incidence countries (150-250/100000) the yield was 166/100000. In our study, seven out of nine TB cases had CXR pathology; however, only one had infiltrations with cavity formation, while the remaining had minor and non-specific findings. Low-dose CXR in pregnancy is considered harmless [33], but the yield with CXR screening in all pregnant women from high-incidence countries (>100/100000), regardless of symptoms and QFT result, would be less cost-effective.

Nine HIV-negative pulmonary TB cases were detected, corresponding to a high incidence of 215/100000 screened pregnant women yearly. Only one had been in contact with healthcare due to suggestive TB symptoms and this patient was the only case which was smear microscopy positive, i.e. highly contagious. This suggests that symptoms of TB disease during pregnancy are either mild or absent which emphasises the importance of active questioning of symptoms and TB exposure, as well as a liberal sputum sampling for M. tuberculosis verification including culture. This is supported in a recent study from south of Sweden by Bullarbo et al. [34], in which only one active TB case out of 902 screened pregnant women was detected due to symptoms.

\section{Conclusions}

Systematic TB screening of pregnant women from high-endemic countries was a feasible complement to regular asylum screening in Stockholm. The cascade of care revealed a high yield of previously unknown LTBI and mostly asymptomatic active TB. Information and optimised routines improved referral to specialised care. LTBI treatment completion was very high. Our findings justify TB screening of this risk group for early detection and treatment.

Conflict of interest: None declared.

Support statement: This work was supported by Stockholms Läns Landsting (grant: Dnr K090-2014) and Karolinska Institutet (grant: Dnr K090-2014). Funding information for this article has been deposited with the Crossref Funder Registry.

\section{References}

$1 \quad$ Yip L, McCluskey J, Sinclair R. Immunological aspects of pregnancy. Clin Dermatol 2006; 24: 84-87.

2 Kidd P. Th1/Th2 balance: the hypothesis, its limitations, and implications for health and disease. Altern Med Rev 2003; 8: 223-246.

3 Singh N, Perfect JR. Immune reconstitution syndrome and exacerbation of infections after pregnancy. Clin Infect Dis 2007; 45: 1192-1199.

4 Cheng VC, Woo PC, Lau SK, et al. Peripartum tuberculosis as a form of immunorestitution disease. Eur J Clin Microbiol Infect Dis 2003; 22: 313-317.

$5 \quad$ Loto OM, Awowole I. Tuberculosis in pregnancy: a review. J Pregnancy 2012; 2012: 379271

6 Malhame I, Cormier M, Sugarman J, et al. Latent tuberculosis in pregnancy: a systematic review. PLoS One 2016; 11: $\mathrm{e} 0154825$.

7 Sobhy S, Babiker Z, Zamora J, et al. Maternal and perinatal mortality and morbidity associated with tuberculosis during pregnancy and the postpartum period: a systematic review and meta-analysis. BJOG 2017; 124: 727-733.

8 Bates M, Ahmed Y, Kapata N, et al. Perspectives on tuberculosis in pregnancy. Int J Infect Dis 2015; 32: 124-127.

9 Zenner D, Kruijshaar ME, Andrews N, et al. Risk of tuberculosis in pregnancy: a national, primary care-based cohort and self-controlled case series study. Am J Respir Crit Care Med 2012; 185: 779-784.

10 Mathad JS, Gupta A. Tuberculosis in pregnant and postpartum women: epidemiology, management, and research gaps. Clin Infect Dis 2012; 55: 1532-1549.

11 Lamb GS, Starke JR. Tuberculosis in infants and children. Microbiol Spectr 2017; 5: 10.1128/microbiolspec.TBTB20005-2015

12 WHO. Implementing The End TB strategy: The Essentials. 2015. https://www.who.int/tb/publications/2015/The Essentials to End TB/en/

13 WHO: Latent TB Infection : Updated and consolidated guidelines for programmatic management. In.; 2018. https://www.who.int/tb/publications/2018/latent-tuberculosis-infection/en/.

14 WHO. Guidelines on the Management of Latent Tuberculosis Infection. WHO, 2015. https://www.who.int/tb/ publications/latent-tuberculosis-infection/en/

15 Public Health Agency of Sweden: Recommendations on preventive measures against latent tuberculosis. 2017, 00497-2017. https://www.folkhalsomyndigheten.se/publicerat-material/publikationsarkiv/r/rekommendationer-forpreventiva-insatser-mot-tuberkulos-halsokontroll-smittsparning-och-vaccination/ 
16 Center for Epidemiology and Community Medicine, Stockholm County Council, 2017: Health examinations of asylum seekers. Report 2017:4. http://dok.slso.sll.se/CES/FHG/Folkhalsoarbete/Rapporter/halsoundersokningar-forasylsokande-och-nyanlanda.pdf

17 LaCourse SM, Cranmer LM, Matemo D, et al. Effect of pregnancy on interferon gamma release assay and tuberculin skin test detection of latent TB infection among HIV-infected women in a high burden setting. J Acquir Immune Defic Syndr 2017; 75: 128-136.

18 Konig Walles J, Tesfaye F, Jansson M, et al. Performance of QuantiFERON-TB Gold Plus for detection of latent tuberculosis infection in pregnant women living in a tuberculosis- and HIV-endemic setting. PLoS One 2018; 13: e0193589.

19 Mathad JS, Bhosale R, Sangar V, et al. Pregnancy differentially impacts performance of latent tuberculosis diagnostics in a high-burden setting. PLoS One 2014; 9: e92308.

20 Pai M, Zwerling A, Menzies D. Systematic review: T-cell-based assays for the diagnosis of latent tuberculosis infection: an update. Ann Intern Med 2008; 149: 177-184.

21 Sarrazin H, Wilkinson KA, Andersson J, et al. Association between tuberculin skin test reactivity, the memory CD4 cell subset, and circulating FoxP3-expressing cells in HIV-infected persons. J Infect Dis 2009; 199: 702-710.

22 Spyridis NP, Spyridis PG, Gelesme A, et al. The effectiveness of a 9-month regimen of isoniazid alone versus 3 and 4-month regimens of isoniazid plus rifampin for treatment of latent tuberculosis infection in children: results of an 11-year randomized study. Clin Infect Dis 2007; 45: 715-722.

23 McClintock AH, Eastment M, McKinney CM, et al. Treatment completion for latent tuberculosis infection: a retrospective cohort study comparing 9 months of isoniazid, 4 months of rifampin and 3 months of isoniazid and rifapentine. BMC Infect Dis 2017; 17: 146.

24 Department of Communicable Diseases Control and Prevention, Stockholm County Council: Screening of pregnant women in Stockholm County. 2019. https://vardgivarguiden.se/globalassets/kunskapsstod/smittskydd/ tuberkulos/screening-av-gravida.pdf.

25 Jonsson J, Westman A, Bruchfeld J, et al. A borderline range for Quantiferon Gold In-Tube results. PLoS One 2017; 12: e0187313.

26 Pareek M, Greenaway C, Noori T, et al. The impact of migration on tuberculosis epidemiology and control in high-income countries: a review. BMC Med 2016; 14: 48.

27 Greenaway C, Pareek M, Abou Chakra CN, et al. The effectiveness and cost-effectiveness of screening for active tuberculosis among migrants in the EU/EEA: a systematic review. Euro Surveill 2018; 23: doi: 10.2807/1560-7917. ES.2018.23.14.17-00542.

28 Alsdurf H, Hill PC, Matteelli A, et al. The cascade of care in diagnosis and treatment of latent tuberculosis infection: a systematic review and meta-analysis. Lancet Infect Dis 2016; 16: 1269-1278.

29 Kulane A, Ahlberg BM, Berggren I. "It is more than the issue of taking tablets": the interplay between migration policies and TB control in Sweden. Health Policy 2010; 97: 26-31.

30 Shedrawy J, Jansson L, Röhl I, et al. Quality of life of patients on treatment for latent tuberculosis infection: a mixed-method study in Stockholm, Sweden. Health Qual Life Outcomes 2019; 17: 158.

31 Norrby M, Wannheden C, Ekstrom AM, et al. Incidence of tuberculosis and the need of prophylactic treatment in persons living with HIV in Stockholm during the era of anti-retroviral therapy 1996-2013. Infect Dis (Lond) 2018; 50: 807-816.

32 Kunst H, Burman M, Arnesen TM, et al. Tuberculosis and latent tuberculous infection screening of migrants in Europe: comparative analysis of policies, surveillance systems and results. Int J Tuberc Lung Dis 2017; 21: 840-851.

33 Vock P. Clinical perspective on diagnostic X-ray examinations of pregnant patients - What to take into account. Phys Med 2017; 43: 165-171.

34 Bullarbo M, Barnisin M, Vukas Radulovic N, et al. Low prevalence of active tuberculosis among high-risk pregnant and postpartum women in Sweden: a retrospective epidemiological cohort study using and evaluating TST as screening method. Infect Dis Obstet Gynecol 2018; 2018: 3153250. 